\title{
Understanding Imaging and Energy-loss Spectra Due to Phonon Excitation
}

\author{
L.J. Allen ${ }^{1,2}$, B.D. Forbes ${ }^{1}$, S.D. Findlay ${ }^{3}$, H.G. Brown ${ }^{3}$ and N.R. Lugg ${ }^{4}$ \\ 1. School of Physics, University of Melbourne, Melbourne, Australia. \\ 2. Ernst Ruska Centre for Microscopy and Spectroscopy with Electrons, Research Centre Jülich, Jülich, \\ Germany. \\ 3. School of Physics and Astronomy, Monash University, Melbourne, Australia. \\ 4. Crystal Interface Laboratory, Institute of Engineering Innovation, University of Tokyo, Tokyo, Japan.
}

Recent developments have improved the attainable energy resolution in electron energy-loss spectroscopy in aberration-corrected transmission electron microscopy to the order of $10 \mathrm{meV}$ [1]. In principle, this allows imaging and spectroscopy of crystals using the phonon sector of the energy-loss spectrum at atomic resolution, a supposition supported by further recent work [2].

The "quantum excitation of phonons" model [3] encapsulates the physics necessary to simulate the atomic resolution imaging of crystals based on phonon excitation. Figure 1(a) shows the distribution of thermally scattered electrons with a scanning transmission electron microscopy (STEM) probe placed above various columns of atoms in strontium titanate and Fig. 1(b) shows line scan images across strontium and titanium/oxygen columns in $<001>$ strontium titanate, showing atomic resolution imaging is possible even for electrons scattered through small angles (less than $20 \mathrm{mrad}$ ) [4].

A fundamental theory to calculate the phonon-loss sector of the energy-loss spectrum for electrons scattered from crystalline solids can be formulated based on transition potentials [5]. We use a correlated model for the atomic motion for calculating the vibrational modes of a system. This allows spectra to be calculated for crystalline silicon, illuminated by a plane wave, as shown in Fig. 2 (a). Also shown in Fig. 2(a) is the spectrum calculated in an Einstein model, in which atoms in the specimen are assumed to vibrate independently. Whilst the details are quite different the spectra integrate to the same value. When using an atomic-scale, focused coherent probe, the shape of the spectra obtained depend on probe position and also on the size of the spectrometer aperture [5]. Spectra calculated in an Einstein model can be related to a classical view [6] of the energy-loss process, as shown in Fig. 2(b).

The phenomenon of "aloof beam" imaging [7-10] can be explained within the context of the transition potential model. The fall off in the intensity of the signal, as a function of the distance of a point probe from the specimen, can be investigated [11].

\section{References:}

[1] OL Krivanek et al, Nature 514 (2014) p. 209.

[2] C Dwyer et al, Phys. Rev. Lett. 117 (2016) 256101.

[3] BD Forbes et al, Phys. Rev. B 82 (2010) 104103.

[4] NR Lugg et al, Phys Rev. B 91 (2015) 144108.

[5] BD Forbes et al, Phys Rev. B 94 (2016) 014110.

[6] G Argentero et al, Ultramicroscopy 151 (2015) p. 23.

[7] A Howie, Ultramicroscopy 11 (1983) p. 141.

[8] P Rez, Microsc. Microanal. 20 (2014) p. 671. 
[9] RF Egerton, Ultramicroscopy 159 (2015) p. 95.

[10] PA Crozier et al, Ultramicroscopy 169 (2016) p. 30.

[11] This research was supported under the Australian Research Council's Discovery Projects funding scheme (Projects DP110102228 and DP140102538). N.R.L. was supported by the Japan Society for the Promotion of Science (JSPS).
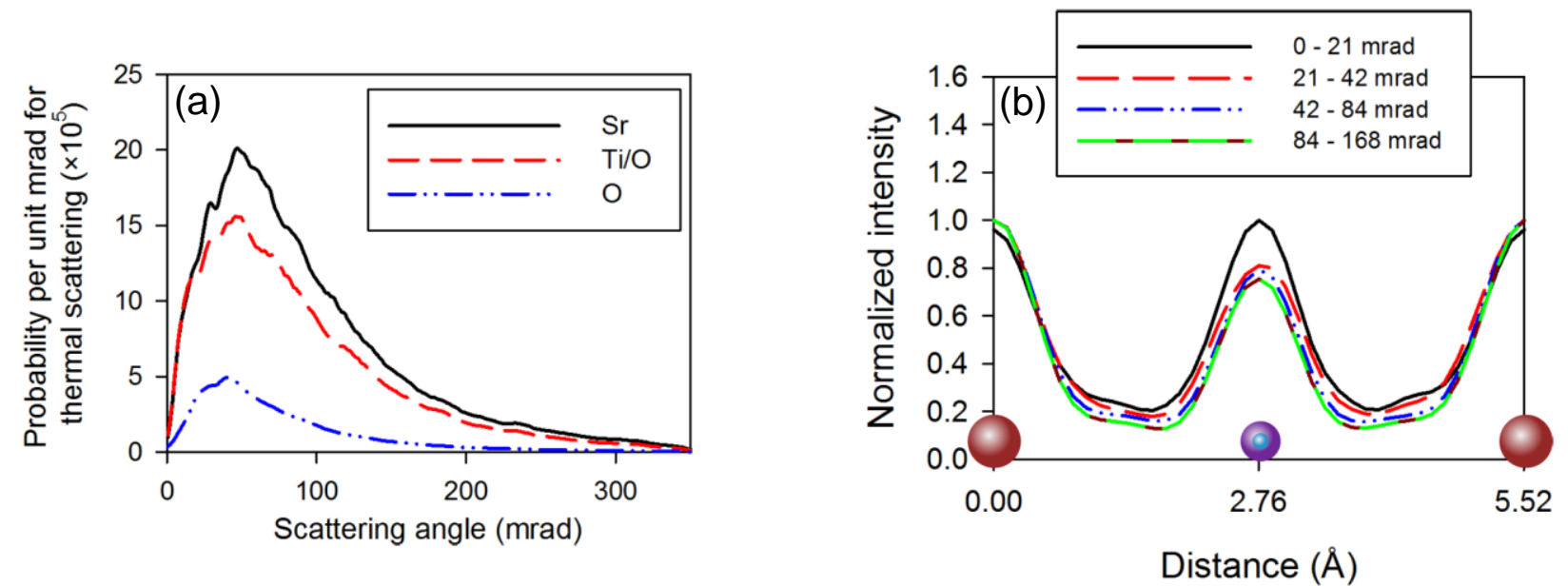

Figure 1. (a) Probability density for the thermally scattered intensity in the diffraction plane as a function of scattering angle for an unaberrated convergent electron beam of accelerating voltage $120 \mathrm{kV}$ and convergence semiangle 21 mrad incident upon a $200 \AA$ thick specimen of $<001>$ strontium titanate. The probe has been placed above strontium, titanium/oxygen, and oxygen columns. (b) STEM intensity profiles for thermally scattered electrons taken across strontium and titanium/oxygen columns for a specimen thickness of $200 \AA$. Each profile has the maximum value normalized to unity. Large (brown) circles indicate columns of strontium atoms, the medium (purple) circle and the small (blue) inlaid circle indicate the presence of titanium atoms and oxygen atoms in the same column.
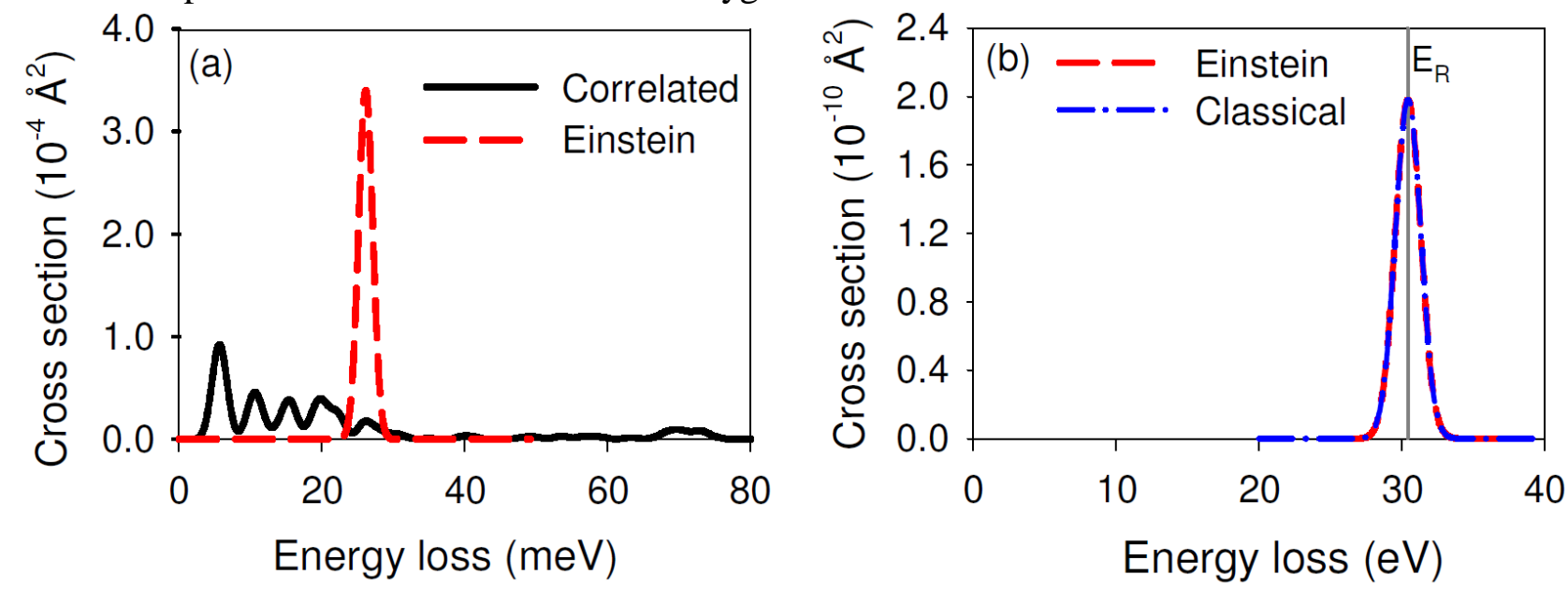

Figure 1. Phonon-loss spectra for $300 \mathrm{keV}$ plane-wave electrons incident along the [001] zone axis in silicon, calculated at room temperature. (a) Spectrum obtained for single-phonon excitation in both the correlated and Einstein models, which is dominated by scattering in the forward direction. (b) Spectrum in the strictly backwards direction for the Einstein model, taking into account multiphonon excitation, and a classical model incorporating Doppler broadening. The peak value occurs at the classical recoil energy $E_{R}$, indicated by the solid line. The Einstein and classical model results in panel (b) have been normalized to each other and are almost indistinguishable. 